\title{
1 A cell-free synthetic biochemistry platform for raspberry ketone production
}

2

3 Simon J Moore ${ }^{1,3}$

4 Tomasso Tosi $^{3}$

5 David Bell ${ }^{1,3}$

6 Yonek B Hleba ${ }^{1,2}$

$7 \quad$ Karen M Polizzi $i^{1,2}$

8 Paul S Freemont ${ }^{1,3}$

9

$10{ }^{1}$ Centre for Synthetic Biology and Innovation, Imperial College London, South

11 Kensington Campus, Exhibition Road, London, SW7 2AZ, UK

$12{ }^{2}$ Department of Life Sciences, Imperial College London, South Kensington Campus,

13 Exhibition Road, London, SW7 2AZ, UK

$14{ }^{3}$ Department of Medicine; Imperial College London, South Kensington Campus,

15 Exhibition Road, London, SW7 2AZ, UK

16

17 Keywords

18 Synthetic biology, fine chemical, raspberry ketone, polyketide synthase, synthetic

19 biochemistry 


\section{Abstract}

23 Cell-free synthetic biochemistry provides a green solution to replace traditional

24 petroleum or agricultural based methods for production of fine chemicals. 4-(4-

25 hydroxyphenyl)-butan-2-one, also known as raspberry ketone, is the major fragrance

26 component of raspberry fruit and is utilised as a natural additive in the food and

27 sports industry. Current industrial processing standards involve chemical extraction

28 with a yield of 1-4 mg per kilo of fruit. As such its market price can fluctuate up to

$29 \$ 20,000$ per $\mathrm{kg}$. Metabolic engineering approaches to synthesise this molecule by

30 microbial fermentation have only resulted in low yields of up to $5 \mathrm{mg} \mathrm{L}^{-1}$. In contrast,

31 cell-free synthetic biochemistry offers an intriguing compromise to the engineering

32 constraints provided by the living cell. Using purified enzymes or a two-step semi-

33 synthetic route, an optimised pathway was formed for raspberry ketone synthesis

34 leading up to $100 \%$ yield conversion. The semi-synthetic route is potentially scalable

35 and cost-efficient for industrial synthesis of raspberry ketone. 
Introduction

38 For fine chemical biomanufacturing, synthetic biology aims to provide green solutions to replacing traditional petroleum or arable farming based production methods ${ }^{1-6}$. The synthetic biochemistry approach, whereby metabolic pathways can be entirely reconstituted within a test-tube with purified enzymes ${ }^{7-11}$ or cell-free extracts ${ }^{12-14}$, offers a realistic concept to traditional cell based engineering, with the potential for high performance synthesis of fine chemicals and recombinant proteins to the industrial scale ${ }^{11,15}$.

4-(4-hydroxyphenyl)butan-2-one, commonly referred to as raspberry ketone, is the major fragrance compound from raspberry berries (Rubeus rubrum). Raspberry ketone is harvested by chemical extraction at a ratio of 1-4 mg per kilo of fruit ${ }^{16}$. This presents a highly-inefficient purification process, which is reflected by a high market price for naturally extracted raspberry ketone that fluctuates at approximately $\$ 10,000-20,000 \mathrm{~kg}^{-1}$, with global production estimated at between $100-200$ tons per annum ${ }^{16,17}$. As an alternative green solution for its production, raspberry ketone can be synthesised in microbial cells. However, current efforts in either Escherichia coli ${ }^{17}$ or Saccharomyces cerevisiae ${ }^{17,18}$ have only produced limited quantities of raspberry ketone (from 0.2 to $5 \mathrm{mg} \mathrm{L}^{-1}$ ), even despite the use of high cell-density fermentation. The raspberry ketone biosynthetic pathway in plants begins from tyrosine or phenylalanine and belongs to the flavonoid natural product family, which includes the medicinal compounds curcumin ${ }^{19,20}$, naringenin ${ }^{21}$, resveratrol ${ }^{22}$ and gingerole ${ }^{23,24}$. The synthesis of flavonoid natural products requires a dedicated type III polyketide synthase that uses the cofactor malonyl-CoA for chain extension. For raspberry 61 ketone, the enzyme responsible for this step is the benzalacetone synthase (BAS), 
which converts p-coumoryl-CoA and malonyl-CoA into 4-hydroxybenzalacetone (HBA) using a unique decarboxylation event ${ }^{25}$, thus affording the precursor of raspberry ketone. Possible limitations for engineering raspberry ketone in cells include a limited malonyl-CoA pool ( $35 \mu \mathrm{M}$ in glucose catabolism) in $E$. coli ${ }^{26,27}$ and a comparatively slow turnover rate $\left(0.1 \mathrm{~s}^{-1}\right)$ for the BAS enzyme ${ }^{28}$. Additionally, previous metabolic engineering studies have led to a mixed yield of raspberry ketone and its precursor HBA, which requires a further double bond reduction. Therefore, an efficient alkene reductase is also required to complete the pathway.

Routinely in synthetic biology, microbial cells are engineered through plasmid or chromosomal based heterologous gene expression to synthesise a desired synthetic fine chemical. However, this often presents a myriad of challenges to the living cell. This includes, but is not limited to, cellular burden ${ }^{29}$, metabolic flux ${ }^{30}$, accumulation of toxic intermediates ${ }^{4,31}$, poor substrate availability ${ }^{26}$ and non-productive chemical or enzyme based side-reactions ${ }^{32}$. In addition, whilst in vitro deduced enzyme $k_{\text {cat }}$ values are often reflective of in vivo catalytic rates $\left(k_{\mathrm{app}}\right)$, it should also be considered that the cellular environment can modulate enzyme controlled pathways through changes in thermodynamics or substrate availability ${ }^{33}$. The low yields of raspberry ketone obtained in microbial cells ${ }^{17,18}$ suggests that an enzyme bottleneck or product toxicity limits it's production in vivo.

For fine chemical pathways, as well as drugs and recombinant proteins, an in vitro approach offers a unique opportunity to the biochemist to control and modify synthetic pathways outside the regulatory control of the cell. Using raspberry ketone as a model pathway, we detail a purified enzyme approach to achieve a high-yield 
87 synthesis in a one-pot reaction. Together with other recent synthetic biochemistry 88 studies ${ }^{11,13}$, we feel this approach is potentially expandable to other high-value fine

89 chemicals that require cost efficient cofactor regeneration (i.e. malonyl-CoA, ATP) for

90 in vitro synthesis. In summary, we present a modularised enzyme prototyping 91 approach to completely convert the substrate tyrosine via a five-enzyme cascade 92 into raspberry ketone, as well as demonstrating how enzyme levels competing for 93 the key cofactor, coenzyme $\mathrm{A}(\mathrm{CoA})$, require balancing for optimal performance. To 94 complement these findings, we also present a crystal structure of the NADPH95 dependent raspberry ketone reductase, which we use to relax its cofactor specificity 96 towards NADH utilisation for an improved low-cost in vitro engineering. 
Results

\section{Enzyme synthesis of raspberry ketone pathway in vitro}

100 In order to begin studying the raspberry ketone pathway in vitro and identify key

101 metabolic bottlenecks, we selected a synthetic five-step pathway for raspberry

102 ketone using a combination of bacterial, fungi and plant enzymes (Figure 1A). The starting point for this pathway begins from tyrosine, the natural substrate for raspberry ketone synthesis in plants, using tyrosine ammonia lyase (TAL) to deaminate tyrosine, forming $p$-coumarate ${ }^{34}$. This in turn is activated for malonyl-CoA extension by the addition of CoA by an ATP-dependent CoA ligase (PCL) to form $p$ coumoroyl-CoA ${ }^{35}$. Following this, the benzalacetone synthase (BAS) uses malonylCoA for chain extension via a double decarboxylation event to release the HBA product ${ }^{25,28}$. Finally, a NADPH-dependent raspberry ketone synthase (RKS), a

110 double bond reductase, converts HBA into raspberry ketone ${ }^{36}$. Based on this 111 synthetic pathway, a set of highly active and kinetically well-characterised enzymes 112 for TAL, PCL and a malonyl-CoA synthetase (MatB) were selected from the 113 BRENDA database (see materials and methods), together with BAS and RKS

114 (previously abbreviated as RZS for raspberry ketone/zingerone synthase), where

115 only a single enzyme has been reported and characterised ${ }^{25,36}$. Initially, each 116 enzyme was recombinantly produced in E. coli BL21 Gold (DE3) pLysS and purified

117 at high-yields $\left(\sim 10-100 \mathrm{mg} \mathrm{L}^{-1}\right)$ from the soluble fraction with at least $95 \%$ purity as 118 estimated by SDS-PAGE (Figure 1B). The activity of TAL and PCL enzymes was 119 also assayed individually (Supplementary Figure S1) and results were in close 120 agreement to previous reported literature values ${ }^{37,38}$. 
122 To test the synthetic in vitro pathway, $5 \mu \mathrm{M}$ of purified TAL, PCL, MatB, BAS and

123 RKS enzymes were incubated with $1 \mathrm{mM}$ tyrosine and substrates/cofactors (MgATP,

124 CoA, malonate and NADPH). Please refer to the materials and methods for further

125 details. Samples were removed at time points and quantified by reverse phase (C18)

126 liquid-chromatography mass spectrometry (LC-MS) (Figure 1C). To begin, tyrosine is

127 steadily depleted, whilst $p$-coumarate accumulates up to a maximum of $110 \mu \mathrm{M}$.

128 Under these conditions, the HBA intermediate is not detectable, with the RKS fully

129 converting its substrate into raspberry ketone. Raspberry ketone synthesis occurred

130 at an initial linear rate of $0.63 \mu \mathrm{M} \mathrm{m^{-1 }}$ for up to 6 hours, with a $33.7 \%$ yield (337

$131 \mu \mathrm{M})$ achieved at end-point, which is equivalent to $55 \mathrm{mg} \mathrm{L}^{-1}$ under batch synthesis.

132 This suggested that the synthetic pathway was highly active in relative comparison to

133 previous low yields achieved from cell-based metabolic engineering efforts (from

$1340.28 \mathrm{mg} \mathrm{L}^{-1}$ and $5 \mathrm{mg} \mathrm{L}^{-1}$ in yeast and $E$. coli, respectively) ${ }^{17,18}$.

136 A fluorescence biosensor for optimising flavonoid synthesis in vitro

137 Following the initial demonstration of raspberry ketone synthesis in vitro, we next sought to improve the overall pathway performance by identifying key bottlenecks and optimal pathway conditions. Due to the presence of an extra double bond, the

140 product of the BAS enzyme, HBA, shares broad UV-Visible absorbance with the

141 precursor intermediates $p$-coumarate and $p$-coumoroyl-CoA. Therefore, it was not

142 possible to rapidly optimise the pathway with respect to BAS activity using a UV-

143 Visible absorbance assay. Instead, the pathway was modularised by designing a

144 fluorescence-based product detection assay to separately optimise $p$-coumoryl-CoA

145 and malonyl-CoA synthesis, the shared substrates for the BAS enzyme (Figure 2A) 
146 with the rationale that a delicate balance of these is likely necessary to maximise

147 total production concentration because they both rely on CoA for their synthesis.

149 Previously, curcumin, which is a structural dimer of raspberry ketone, was identified

150 to exhibit fluorescence when bound non-specifically to Gram-negative bacterial 151 extracellular curli (amyloid-type) protein fibres ${ }^{39}$. The precursor of curcumin, bidesmethoxycurcumin (BDMC), is synthesised from p-coumoroyl-CoA and malonyl-

153 CoA by the curcumin synthase (CUS) ${ }^{40}$, a type III polyketide synthase that is related

154 to the BAS enzyme with $69 \%$ amino acid identity. Additionally, a NADPH-dependent

155 curcumin double bond reductase (CurA), which is related to the RKS reductase 156 enzyme with $38 \%$ amino acid identity, was previously characterised from E. coli ${ }^{41}$. A

157 Phyre ${ }^{42}$ structural model of CurA suggested a large active site dominated by Tyr and Phe residues. Based on the potential fluorescence properties of non-specifically bound curcumin, we speculated that substrate binding of the apo-form of CurA to

160 either curcumin or BDMC would also generate fluorescence through $\pi-\pi$ stacking within the aromatic active site. To test this, we overexpressed and purified CurA with an N-terminal His 6 -tag from E. coli BL21 Gold (DE3) pLysS. Mixing an equal amount

$163(10 \mu \mathrm{M})$ of apo-CurA with curcumin resulted in strong fluorescence with a maximum excitation and emission peaks at $425 \mathrm{~nm}$ and $520 \mathrm{~nm}$, respectively, indicating ligand binding (Supplementary Figure S2). If an excess $(100 \mu \mathrm{M})$ of NADPH was also

166 added, the yellow coloured curcumin was rapidly reduced into the colourless

167 tetrahydrocurcumin. This resulted in a quenching of the fluorescence, due to double bond reduction and a loss of conjugation. This suggested that CurA could bind curcumin in the absence of NADPH and therefore does not require the formation of a

170 ternary complex. Next, with $25 \mu \mathrm{M}$ of CurA incubated with an increasing 
concentration of curcumin $(0-100 \mu \mathrm{M})$, the fluorescence signal fitted an exponential saturation curve (Supplementary Figure S3). Further increases in signal could be achieved with higher levels of CurA protein, but since the yield of recombinant CurA in $E$. coli was limiting $\left(\sim 10 \mathrm{mg} \mathrm{L}^{-1}\right)$, a maximum level of $25 \mu \mathrm{M}$ was used for subsequent assays to preserve protein stocks.

177 To confirm the use of this protein-ligand sensor as a real-time fluorescence assay of

$178 \mathrm{BDMC/curcumin}$ synthesis, $25 \mu \mathrm{M}$ of CurA was mixed in a one-pot reaction containing $1 \mu \mathrm{M}$ of TAL, PCL, MatB and the type III polyketide synthase, CUS, along

180 with the necessary cofactors and $1 \mathrm{mM}$ Tyrosine. In addition, each enzyme was

181 individually omitted as a control to confirm the specificity of the assay. Following a 182 time-course reaction, an increasing fluorescence signal was observed 183 (Supplementary Figure S4), relative to a stable background signal if any of the 184 enzymes or substrates were omitted (Supplementary Figure S4). The initial rate of 185 the fluorescence signal was further improved by increasing the concentration of the substrate tyrosine or the CUS enzyme (Supplementary Figure S5). Furthermore, if a $1 \mu \mathrm{M}$ aliquot of $\mathrm{NADPH}$ was injected midway through the reaction, a reduced

188 fluorescence was observed, confirming that reduction of the double bond quenches 189 the fluorescence. This confirmed the sensitivity and specificity of the assay for 190 BDMC synthesis.

193 To potentially improve the activity of the BAS enzyme, we next sought to use our 194 novel fluorescence small molecule sensor to rapidly optimise the synthesis of the 195 substrates $p$-coumoryl-CoA and malonyl-CoA in vitro. Firstly, one-pot synthesis of 
196 BDMC accumulated a yellow visual appearance (Figure 2C), which was fluorescent

197 in the presence of the CurA sensor (Figure 2D). Initially, the conditions for in vitro synthesis of BDMC were optimised. Firstly, tyrosine and CUS levels were set at 5

$199 \mathrm{mM}$ and $25 \mu \mathrm{M}$, respectively, so that these parameters were not rate limiting

200 (Supplementary Figure S5). An important consideration for in vitro enzyme systems

201 is the concentration of substrates and cofactors (Figure 2B). BDMC synthesis was

202 next optimised with respect to ATP, $\mathrm{Mg}^{2+}, \mathrm{CoA}$ and malonate levels. As expected,

203 the ATP level was critical to BDMC synthesis with at least $1 \mathrm{mM}$ required to reach

204 fluorescence saturation ( $65,000 \mathrm{RFU})$, whilst for optimal malonyl-CoA supply, a concentration of greater than $2.5 \mathrm{mM}$ malonate was required for maximal activity (>60,000 RFU) and fluorescence saturation. The $\mathrm{Mg}^{2+}$ levels were equally optimal at

0.1-1 mM, but with higher levels, a biphasic fluorescence curve was observed

(Figure 2B). A similar observation also occurred with the levels of CoA, where concentrations below the optimum level $(0.25 \mathrm{mM})$ were also found to demonstrate a biphasic response. One potential interpretation of this response is a temporary depletion of free CoA availability, thus leading to an imbalance between $p$-coumoryl-

212 CoA and malonyl-CoA levels for the CUS enzyme. In contrast, if higher levels of CoA (>1 mM) were added, complete inhibition of CurA-BDMC was observed (Figure 2B).

215 We next used this optimised set of conditions to determine if the enzyme levels of

216 TAL, PCL or MatB control BDMC synthesis. To do this, each enzyme was varied in a

217 4-fold dilution series from 0.0156 to $16 \mu \mathrm{M}$. Firstly, some background fluorescence

218 attributed to high levels of p-coumoryl-CoA occurred if the level of the PCL enzyme 219 was increased above $1 \mu \mathrm{M}$ (Figure 2E). However, if both the levels of the TAL and 220 PCL enzymes were optimised to a peak concentration of $16 \mu \mathrm{M}$ and $1 \mu \mathrm{M}$, 
respectively, maximal BDMC synthesis $(87,400 \mathrm{RFU})$ was reached. Interestingly, by varying PCL and MatB together, noticeably a careful balance of enzymes was required to prevent pathway inhibition, possibly through depletion of free CoA (Figure

2D). This evidence overall suggests that the pathway is tightly regulated by a

sensitive interplay between the free CoA pool, CoA derivatives, CoA-dependent enzymes and the rate-limiting activity of the type III polyketide synthase (CUS). Therefore, whilst this experiment only provides a relative measure of pathway activity, it demonstrates that a finely tuned interplay between enzyme levels and the CoA cofactor is required for optimal pathway performance. This observation would

230 not be possible to detect through cell-based engineering. Based on these 231 measurements, we next used the optimal parameters to determine whether raspberry ketone synthesis could be improved.

\section{Raspberry ketone biosynthesis is inhibited by a high tyrosine concentration}

We next sought to increase the yield of raspberry ketone synthesis in vitro by providing optimised levels of $p$-coumoroyl-CoA and malonyl-CoA synthesis using the parameters determined in CurA-BDMC fluorescence assay. Firstly, batch reactions were prepared with varying concentrations of tyrosine from 100-1000 $\mu \mathrm{M}$. Between $100-300 \mu \mathrm{M}$ tyrosine, raspberry ketone was produced at a $100 \%$ yield (Figure $3 \mathrm{~A}$ ). Beyond this, the level of $p$-coumarate rises, without a further increase in raspberry ketone yield. Initially, we suspected that the supply of malonyl-CoA or $p$-coumoryl-

242 CoA was limited by the exhaustion of the ATP supply. However, although providing

243 ATP regeneration through phosphoenolpyruvate (PEP) and pyruvate kinase increased the flux of tyrosine and $p$-coumarate, the net yield of raspberry ketone was 
$50 \%$ ) of the BAS catalysed side-product bisnoryanogonin (BNY), in comparison to standard conditions where only minor levels $(\sim 5 \%)$ were detected by LC-MS. BNY is

a side-product of the BAS enzyme that is produced by an additional malonyl-CoA extension of the diketide intermediate ${ }^{43}$. Previously, BNY was produced only at

250 minor levels at mildly alkaline $\mathrm{pH}(7.5-8.5)$, but elevated at $\mathrm{pH} 6.0-7.5$ in Tris- $\mathrm{HCl}$ or 251 phosphate buffers ${ }^{43}$. To attempt to correct this issue, we repeated the synthesis of raspberry ketone in a range of alkaline buffers $(\mathrm{pH} 7.5-9.0)$ and as a time-course at $\mathrm{pH} 9$ (Supplementary Figure S7). Increasing the $\mathrm{pH}$ favoured the activities of the TAL and PCL enzymes, but this did not further increase the levels of raspberry ketone and only a minor decrease in the levels of the BNY side-product was observed. Under these forced synthetic conditions, it appears that the HBA synthesis activity of the BAS enzyme switches towards a preference of BNY synthesis. Since we could not find a route to eliminate BNY synthesis and this capped our overall yield of raspberry ketone, we next considered how the cost-efficiency of this in vitro pathway could be improved by relaxing the cofactor specificity of the NADPHdependent RKS reductase step.

\section{NADH is rate limiting for in vitro raspberry ketone synthesis}

264 The final step of raspberry ketone synthesis is catalysed by the NADPH-dependent double bond reductase RKS. For in vitro based biocatalysis, NADH ${ }^{44}$ or biomimetic 266 analogues ${ }^{45}$ are preferred to provide increased stability and reduced cost for 267 reductive enzyme reactions. We therefore revisited the one-pot synthesis of raspberry ketone providing $\mathrm{NADH}$, as opposed to $\mathrm{NADPH}$, as a cofactor

269 (Supplementary Figure S6). With this, whilst the rate of tyrosine deamination by TAL 270 remains unchanged, an increased accumulation of $p$-coumarate $(172 \mu \mathrm{M})$ and HBA 
$271(15 \mu \mathrm{M})$ is observed after 6 hours, with only trace levels of raspberry ketone

272 detected. Unsurprisingly, only trace levels of raspberry ketone were detected during

273 the time-course, whilst after $48 \mathrm{hrs}$, a $7.6 \%(76.1 \mu \mathrm{M})$ yield of raspberry ketone was

274 achieved. The initial lag in raspberry ketone synthesis confirmed that the RKS enzyme had a clear preference for NADPH as a cofactor.

277 To further understand the RKS reductase, the enzyme was next characterised in

278 vitro. Firstly, HBA the natural substrate for RKS is yellow in colouration at $\mathrm{pH}>6$ and strongly absorbs between $250-450 \mathrm{~nm}$, which overlaps with $\mathrm{NAD}(\mathrm{P}) \mathrm{H}$ absorbance at $340 \mathrm{~nm}$ for kinetic characterisation. Instead, to initially obtain kinetic parameters for the RKS enzyme, the substrate analogue phenylbuten-2-one was used since it lacks UV-Visible absorbance at $340 \mathrm{~nm}$. To begin, the RKS enzyme shares sequence similarity ( $77 \%$ amino acid identity) and similar kinetic properties to the previously characterised promiscuous Nicotiana tabacum double bond reductase NtDBR ${ }^{46}$. For example, both RKS and NtDBR share increased activity towards acidic $\mathrm{pH}$ conditions (Supplementary Figure S8). For all further RKS enzyme assays, these were measured at $30^{\circ} \mathrm{C}$ and $\mathrm{pH} 6.4$, with $1 \mathrm{mM}$ of phenylbuten-2-one. Under these conditions, RKS shows an apparent $K_{\text {cat }} / K_{\mathrm{m}}$ of 58 and $1.3 \mathrm{mM} \mathrm{s}^{-1} \mathrm{mM}^{-1}$ with NADPH and NADH, respectively (Supplementary Table S3). This demonstrates a 45-fold preference for NADPH as a cofactor.

\section{Structure guided engineering of a NADH proficient RKS}

293 A number of studies have highlighted that the cofactor specificity of NADPH-

294 dependent reductase enzymes can be relaxed towards NADH utilisation ${ }^{47-50}$ by 
296 preference to the ribose 5 '-hydroxyl group (NADH) ${ }^{51,52}$. To aid in the rational design

297 of increasing NADH activity of the RKS enzyme, we crystallised and solved the 298 tertiary structure complex of RKS with raspberry ketone pathway substrate HBA 299 (Figure 4A) and the cofactor NADPH (Figure 4B), deposited as pdb: 6EOW. By 300 analysing the $2 F_{0}-F_{c}$ Fourier syntheses, two configuration states were observed at a 50:50 ratio due to crystal soaking. The mixed states could be as a result of NADPH and ternary complex formation to the active site, thus displacing any NADP ${ }^{+}$present from crystallisation. In the ternary complex state, additional electron density for HBA binding is observed, with $\pi-\pi$ stacking between the HBA and nicotinamide aromatic rings (Figure 4A) with a hydride transfer distance of $3.06 \AA$ to the alkene double bond. Furthermore, in the ternary complex, an additional patch of electron density consistent of a flexible loop supporting $\mathrm{Y} 72$ is observed. This flexible loop forms a cap over the active site (closed loop), with the para hydroxyl group in HBA moving towards Y72. With respect to HBA binding, the substrate is bent within the active site and is encased by aromatic residues Y59, Y72, Y85, F107, Y263 and F290

311 (Supplementary Figure S9). We speculate that this this closed loop formation holds

312 the HBA within the active site prior to transition state. Interestingly, in contrast to 313 apo-RKS and NtDBR, the Y72 flexible loop lacks electron density and seemingly 314 points away from the active site (open loop state). Finally, in regards to cofactor 315 binding, in both states the $\operatorname{NADP}\left(\mathrm{H} /{ }^{+}\right)$cofactor is bound in a typical conformation as 316 is observed in the previous related structures ${ }^{46,53}$ with the binding specificity 317 provided by a triad of hydrogen bond based contacts with the G191 backbone 318 nitrogen and the side chains K195 and Y211 (Figure 4B). Whilst K212 is also 319 present, it is rather removed from binding to the ribose 5'-phosphate. Importantly, 
K195 can interact with either the ribose 5'-phosphate or neighbouring 4'-hydroxyl group.

In an effort to modify the cofactor specificity of the RKS enzyme, a number of amino acid substitutions at G191 and Y211 were made and steady-state kinetics was determined where appropriate (Figure 4C and Supplementary Table S3). We did not attempt to modify K195 due to its role in bonding to the 4' hydroxyl group found in both NADPH and NADH. We instead chose to modify G191 to provide a short polar side chain ( $D$ and $N$ ) that can provide a hydrogen bond for the 5'-hydroxyl group unique to NADH. Both the D191 and N191 modifications decreased the $k_{\text {cat }} / K_{\mathrm{m}}$ from $58 \mathrm{mM}^{-1} \mathrm{~s}^{-1}\left(\mathrm{RKS}^{\mathrm{WT}}\right)$ with NADPH to 14 and $15\left(\mathrm{~s}^{-1} \mathrm{mM}^{-1}\right)$, respectively (Figure $\left.4 \mathrm{C}\right)$.

331 This decrease in catalytic efficiency was due to an increased $K_{m}$ for NADPH with the variants. Interestingly, for $\mathrm{N} 191$, the $k_{\text {cat }}$ increased by 3 -fold up to $1.07 \mathrm{~s}^{-1}$ in comparison to the wild-type $\left(0.31 \mathrm{~s}^{-1}\right)$. One possible interpretation is that steric

334 hindrance of N191 with the 5' phosphate increases the catalytic rate by enhancing $\mathrm{NADP}^{+}$release through decreasing its binding affinity. In contrast, with $\mathrm{NADH}$ as the cofactor, whilst N191 did not improve NADH utilisation, D191 was found to increase the $k_{\text {cat }} / K_{\mathrm{m}}$ from $1.3 \mathrm{mM}^{-1} \mathrm{~s}^{-1}\left(\mathrm{RKS}^{\mathrm{WT}}\right)$ to $5.9 \mathrm{mM}^{-1} \mathrm{~s}^{-1}$ (Figure $4 \mathrm{D}$ and Supplementary Table S3). In summary, these mutagenesis experiments at the G191 position demonstrated that the negatively charged (D) side chain improves NADH specificity

340 in preference to a positive charged residue $(\mathrm{N})$. Whilst further modifications to the

341 Y211 position were also tested individually or in combination with D191 and N191

342 (data not shown), no further improvements in NADH specificity or $k_{\text {cat }}$ were found, with most modifications leading to a loss of activity with either NADH or NADPH. 
NADH based in vitro synthesis of raspberry ketone from HBA

346 Whilst the kinetics of RKS (wild-type and D191) were not characterised with its

347 natural substrate, HBA, its activity was demonstrated with $\mathrm{NAD}(\mathrm{P}) \mathrm{H}$ cofactor

348 regeneration. Firstly, HBA was synthesised from the inexpensive substrates $p$ -

349 benzaldehyde ( $\$ 203$ per $\mathrm{kg}$ ) and acetic acid ( $\$ 67$ per $\mathrm{kg}$ ) using an aldol

350 condensation reaction under basic conditions to provide a $76 \%$ yield ${ }^{54}$ (Figure $5 \mathrm{~A}$ ).

351 Then, using the thermostable phosphite dehydrogenase (PtxD) mutant Opt12 ${ }^{55}$ for

$352 \mathrm{NAD}(\mathrm{P}) \mathrm{H}$ regeneration from phosphite, we tested the reduction of the HBA substrate

353 at $30^{\circ} \mathrm{C}$ by following a loss of absorbance at $400 \mathrm{~nm}$ (A400). For negative controls, in

354 the absence of the reductase, phosphite or PtxD, the A400 for $1 \mathrm{mM} \mathrm{HBA}$ remained stable over the time-course measured (Figure 5B). With $1 \mathrm{mM} \mathrm{HBA}, 10 \mu \mathrm{M}$ of RKS,

$20 \mathrm{mM}$ phosphite and an excess of PtxD opt12, complete reduction was achieved

with as little as $10 \mu \mathrm{M}$ of NADPH (Figure 5B). Next, to demonstrate the proficiency of the RKS variants $(10 \mu \mathrm{M})$ with both $\mathrm{NADPH}$ and $\mathrm{NADH}$, a time-course reaction was monitored with $1 \mathrm{mM}$ injections of HBA every hour with $0.25 \mathrm{mM} \mathrm{NAD}(\mathrm{P}) \mathrm{H}, 20 \mathrm{mM}$ 360 phosphite and an excess of PtxD opt12 (Figure 5B). For RKS ${ }^{\mathrm{WT}}$, an initial rate of

59.1 and $12.8 \mu \mathrm{M} / \mathrm{min} / \mathrm{mg}$ was observed with $\mathrm{NADPH}$ and $\mathrm{NADH}$, respectively

362 (Supplementary Table S2). In comparison, for $\mathrm{RKS}^{\mathrm{D} 191}$, the rates of reduction with

$363 \mathrm{NADPH}$ and NADH were nearly equivalent at 46.5 and $43.5 \mu \mathrm{M} / \mathrm{min} / \mathrm{mg}$, respectively

364 (Supplementary Table S2). This demonstrated high-yield and efficient synthesis of 365 raspberry ketone with the $\mathrm{RKS}^{\mathrm{D} 191}$ variant providing an elevated and complete 366 turnover $(\sim 100 \%)$ with the inexpensive NADH cofactor. RKS reductase activity was 367 also stable for several days at room temperature (data not shown). 
370 To understand whether the RKS ${ }^{\mathrm{D} 191}$ variant could be used for in vitro synthesis of

371 raspberry ketone with $\mathrm{NADH}$, three time-course reactions were prepared with $1 \mathrm{mM}$

372 tyrosine and optimised enzyme levels and cofactors with either an absence of the

373 reductase or a combination of $\mathrm{RKS}^{\mathrm{WT}} / \mathrm{NADPH}$ or $\mathrm{RKS}^{\mathrm{D} 191 / N A D H}$. Firstly, ATP

374 regeneration was omitted to limit the accumulation of the BNY intermediate. Then,

375 without the RKS reductase, the reaction accumulated HBA at a rate of $0.81 \mu \mathrm{M} \mathrm{min}{ }^{-1}$

376 (Supplementary Table S2), with a maximum yield of $421 \mu \mathrm{M}$ observed after $48 \mathrm{hrs}$,

377 along with $197 \mu \mathrm{M}$ of leftover $p$-coumarate. By adding $20 \mu \mathrm{M} \mathrm{RKS}^{\mathrm{WT}}$ with $0.5 \mathrm{mM}$

378 NADPH along with cofactor recycling with the phosphite dehydrogenase ${ }^{55}$, in

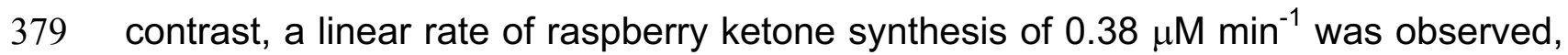

380 whilst after $48 \mathrm{hrs}$, the final concentrations of the intermediates were $291 \mu \mathrm{M} p$ -

381 coumarate and $375 \mu \mathrm{M}$ raspberry ketone, with tyrosine or HBA undetected (Figure

382 6A). The HPLC chromatogram trace suggested that the remaining mixture was

383 composed of the BNY side product. Finally, with $20 \mu \mathrm{M} \mathrm{RKS}{ }^{\mathrm{D} 191}$ introduced with 0.5

$384 \mathrm{mM}$ of NADH and the recycling enzymes, in contrast to its earlier use with the wild-

385 type RKS, the final level of raspberry ketone synthesis was improved to $297 \mu \mathrm{M}$ at a

386 rate of $0.51 \mu \mathrm{M} \mathrm{min}{ }^{-1}$. However, a mixture of $297 \mu \mathrm{M} p$-coumarate and $198 \mu \mathrm{M}$ HBA

387 still remained present (Figure 6B). 


\section{Discussion}

389 Synthetic biochemistry offers an exciting opportunity to design and engineer enzyme

390 pathways outside of the constraints of a living cell. Recent advances include the

391 generation of a synthetic $\mathrm{CO}_{2}$ fixation cycle ${ }^{56}$ and the potential industrial scale

392 synthesis of bioplastic ${ }^{11}$. Here, we show the application of the synthetic biochemistry

393 approach to the synthesis of the natural product raspberry ketone. There is

394 increasing interest in natural product biosynthesis as a source of new antimicrobials,

395 human therapeutics, and fine chemicals. The synthetic biochemistry platform, which

396 allows the rapid optimisation of pathway flux ${ }^{12,57}$ using purified enzymes from

397 different sources to identify high yielding combinations, is a powerful tool for natural

398 product biosynthesis. Potentially, it could be used as a drug discovery tool, e.g. for

399 probing the function of newly identified gene clusters in a sequential manner that

400 allows the documentation of individual enzyme activities.

402 Natural products and other high-value fine chemicals are often only synthesized at

403 very low concentrations in their natural source. Unless chemical logic (i.e reactive species) dictates, metabolic pathway enzymes lack selective pressure to evolve enhanced catalytic rates. For raspberry ketone, only tiny quantities are synthesised in the berries themselves and the process requires weeks of maturation. Therefore,

407 yields as low as 1-4 mg per kilo of raspberry are obtained by chemical extraction of 408 natural raspberry ketone fragrance ${ }^{17}$. To meet the demand of raspberry ketone for 409 the food and sports industry, a two-step organic synthesis via an aldol condensation 410 followed by a rhodium-catalysed hydrogenation reaction is used ${ }^{58}$. However, there is 411 interest in developing a greener approach using synthetic biology to obtain this 
412 natural product. Previous work has investigated synthetically engineered E. coli ${ }^{17}$

413 and Baker's Yeast ${ }^{17,18}$ systems, but yields from fermentation have been limited.

415 One of the advantages of the synthetic biochemistry approach is the ability to 416 produce compounds that are toxic to cells or require high levels of enzyme 417 expression to overcome pathway bottlenecks, which can lead to metabolic burden in 418 vivo. Processes can also be designed to convert central metabolites to products 419 without syphoning off essential resources required for cellular growth. Metabolic 420 engineering in vivo requires a delicate balance between flux towards the product and 421 towards biomass accumulation. When flux towards the product is sufficiently high to 422 reduce cellular growth rates, there is inherent evolutionary pressure to lower productivity because mutations that reduce enzyme expression or primary metabolite usage will lead to a selective advantage. Our synthetic biochemistry platform utilises purified proteins in vitro and therefore is not subject to evolutionary pressure to decrease productivity. In principle, any compound can be produced from any starting material without incurring metabolic burden. Synthetic biochemistry can, therefore, be considered 'evolution-free' and often results in higher yields of product than can be achieved via in vivo production. In our case, we obtained $61 \mathrm{mg} \mathrm{L}^{-1}$ raspberry ketone in a batch system, which is approximately 10-fold higher than the highest

431 reported concentration from fermentation. Further improvement could also be 432 achieved with a continuous flow system such as that reported recently by Hold et al $433 \quad 57$ for active provision of substrates and removal of product. Moreover, during our 434 optimisation experiments, we identified that high concentrations of tyrosine as the 435 starting substrate inhibited the overall yield of raspberry ketone. Our study hints that 436 there may be a novel regulation mechanism of the BAS enzyme, possibly allosteric, 
that prevents over-accumulation of raspberry ketone in its native host. Therefore, synthetic biochemistry can also be a powerful tool for research as well as production of compounds.

Another advantage of the synthetic biochemistry platform is the ability to combine enzymes from different sources with greater ease and less metabolic burden than constructing a strain expressing the whole heterologous pathway. Although we chose to express our enzymes in E. coli, in principle pathway variants that are 'difficult-to-express' or require post-translational modifications can be expressed and purified in another host, but still mixed with an inexpensive cell-lysate derived from $E$. coli to provide energy metabolism to drive the reaction phase. This hybrid approach is not possible with in vivo metabolic engineering where a single production chassis is chosen and its choice may constrain the expression levels of the heterologous pathway or the choice of enzyme source.

452 Raspberry ketone, although one of the simpler polyketides, still has a complicated biosynthetic pathway that requires $A T P$, malonyl-coA, and $N A D(P) H$ as cofactors. We showed that each of these could be recycled in situ through the application of appropriate cofactor regeneration schemes, but that this must be balanced to avoid the accumulation of side products. In addition, we found that the relative levels of malonyl-CoA, $p$-coumoryl-CoA, and free CoA are a key consideration for maximising yield. The use of an in vitro platform allows precise molecular control over the concentration of enzymes, which would be difficult to achieve in vivo with the currently available gene expression tools. 
463 To achieve a fine-tuned in vitro performance, we have also developed a novel

464 protein-ligand binding fluorescence sensor for direct detection of polyketide 465 synthesis activity and used it to maximise the accumulation of the common 466 intermediates of the raspberry ketone and curcumin biosynthesis pathways. This 467 method could be extended for optimisation of biosynthesis of related polyketides or 468 increase flux towards the malonyl-CoA pool in vivo, but will require the inactivation of 469 the CurA reductase activity through point mutations. Given the structural 470 conservation of reductase enzymes, a similar strategy could also be used to develop 471 biosensors for other polyketides in the benzylaklaloid family or to screen variants of 472 the TAL, PCL, or MatB enzymes in order to further increase the yields of raspberry ketone in our synthetic biochemistry platform.

We also demonstrated that raspberry ketone can be synthesised from either tyrosine 476 (the natural substrate in vivo), or through a hybrid chemoenzymatic process where HBA is synthesised in one chemical synthesis step, followed by enzymatic conversion to raspberry ketone. p-coumarate is also an abundant renewable substrate that can be derived from lignin, so is another plausible starting substrate. The chemoenzymatic approach to high-yield raspberry ketone synthesis provides

481 greater simplicity and lower cost. This method is scalable in vitro and potentially also 482 as a microbial fermentation route, potentially providing the most direct method for obtaining industrial yields of raspberry ketone. 
486 Raspberry ketone reductase activity utilises NADPH as a co-substrate which in

487 terms of scalable production is a costly co-factor. To further decrease the cost, we

488 relaxed the cofactor specificity of RKS enzyme to tolerate NADH. A number of

489 studies have highlighted that NADPH-dependent reductases can be engineered to

490 utilise the more stable and inexpensive NADH or biomimetic analogs ${ }^{47-50}$. As a

491 generalised approach, the structure-guided design of $\mathrm{NAD}(\mathrm{P}) \mathrm{H}$ enzymes can be

492 rationalised by altering the affinity for the ribose 5'-phosphate (NADPH) or hydroxyl

493 (NADH) group ${ }^{51,52}$. In the case of the RKS enzyme, position G191 provides flexible

494 control for engineering relaxed cofactor specificity. Its coupled use with an

495 inexpensive phosphate donor via the phosphite dehydrogenase ${ }^{55}$ thus affords a low-

496 cost route to raspberry ketone synthesis in vitro with $\mathrm{NADH}$ using any of the three

497 starting substrates described.

499 Approximately $80 \%$ of the fine chemical market that is used for cosmetics and food

500 additives, is currently produced by oil derived chemical synthesis and thus approved

501 for use if declared as "nature-identical" 59 . For fine chemicals that are extracted naturally from food sources that require large agricultural landmasses, therefore, potentially it is far simpler and more sustainable to engineer greener alternative biocatalytic platforms, either from the use of engineered plants and microbes or

505 through in vitro isolated enzymes. Here, using raspberry ketone as a model pathway,

506 we have demonstrated how an apparently non-productive enzyme pathway can be engineered to a high level of performance from outside of the cell. Essential to this process is the ability to fine tune enzyme pathways in completion, rather than as

509 individual uncoupled kinetics, since shared resources (CoA, ATP) can impact overall 510 yields. In summary, synthetic biochemistry provides an expandable opportunity to 

available under aCC-BY 4.0 International license.

511 design synthetic enzyme ensembles in unison with cofactor availability. We have

512 applied this rational to optimise raspberry ketone, a small molecule that is not easily

513 obtained from within engineered living cells. 


\section{Materials and Methods}

\section{Molecular biology and protein expression}

Routine molecular biology was performed as described previously by our previous research ${ }^{60}$. For the synthetic raspberry ketone pathway the following enzymes were selected - Rhodotorula glutinis TAL 37, Arabidopsis thaliana PCL 35,38, Rhodopseudomonas palustris MatB ${ }^{61}$, Rheum palmatum BAS ${ }^{25,28}$ and Rheum idaeus RKS from ${ }^{36}$. The genes encoding these enzymes were synthesised by ThermoFisher Scientific and codon optimised for E. coli K12 expression with compatibility for EcoFlex cloning ${ }^{60}$. PtxD was used for $N A D(P) H$ recycling from phosphite ${ }^{55}$, whilst ATP regeneration was provided from PEP and rabbit pyruvate kinase (Sigma, UK). All oligonucleotides, plasmids and synthetic DNA sequences are listed in the supporting information. Sequencing was performed by Eurofins, Germany.

\section{Golden Gate Mutagenesis}

We developed a new protocol for mutagenesis based on Golden Gate cloning. Forward and reverse primers were designed for inverse PCR of the plasmid (pTU1A-T7His-RKS-Bba_B0015) to incorporate a Bsal site, which is routinely used in Golden Gate cloning for directional assembly. After digestion with Bsal, the restriction sites are removed, leaving a complementary 4 bp overhang, which is designed to incorporate the mutation site. This anneals and ligates to provide the desired mutation. PCR was performed with the Q5 polymerase (NEB, UK) with 3\% DMSO using the manufacturers standard guidelines. PCR was run on a $1 \%$ agarose gel and the bands were excised and gel purified with a QIAquick Gel Extraction Kit. $20 \mathrm{ng}$ of PCR product was then digest and ligated in a one-pot reaction with $1 \times$ DNA ligase buffer (Promega), 1 unit of Bsal-HF, 5 units of T4 ligase and 1 unit of Dpnl. The reaction was run for 15 cycles at $37^{\circ} \mathrm{C}$ for $5 \mathrm{~min}$ and $16^{\circ} \mathrm{C}$ for $10 \mathrm{~min}$, followed by a further incubation at $50^{\circ} \mathrm{C}$ for $5 \mathrm{~min}$ and $80^{\circ} \mathrm{C}$ for $5 \mathrm{~min}$. $20 \mu \mathrm{L}$ of $\mathrm{DH} 10 \beta$ competent cells were transformed with $2 \mu \mathrm{L}$ of ligation mix and plated onto $100 \mu \mathrm{g} \mathrm{mL}^{-1}$ carbenicillin plates. Single colonies were then sequence verified for incorporation of the mutation site.

\section{Protein expression and purification}

His $_{6}$-tagged recombinant TAL, PCL, BAS, MatB and RKS were over-produced in $E$. coli BL21-Gold (DE3) grown at $37^{\circ} \mathrm{C}, 200 \mathrm{rpm}$ in $2 \mathrm{YT}$ medium with $100 \mu \mathrm{g} / \mathrm{ml}$ ampicillin until an $\mathrm{OD}_{600}$ of 0.6 was reached. Cells were induced with $0.4 \mathrm{mM}$ IPTG and grown overnight at $21^{\circ} \mathrm{C}$ at $200 \mathrm{rpm}$. Cell were collected by centrifugation at $6,000 \times \mathrm{g}, 4^{\circ} \mathrm{C}$ for $20 \mathrm{~min}$, then re-suspended in binding buffer $(20 \mathrm{mM}$ Tris- $\mathrm{HCl} \mathrm{pH}$ $8,500 \mathrm{mM} \mathrm{NaCl}, 5 \mathrm{mM}$ imidazole) and lysed by sonication. Cell-lysates were clarified with centrifugation at $45,000 \times \mathrm{g}, 4^{\circ} \mathrm{C}$ for $20 \mathrm{~min}$ and purified by gravity flow using Ni-NTA agarose (Generon). His 6 -tagged proteins were washed with increasing concentrations of imidazole $(5,30$ and $70 \mathrm{mM})$ in $20 \mathrm{mM}$ Tris- $\mathrm{HCl} \mathrm{pH} 8,500 \mathrm{mM}$ $\mathrm{NaCl}$, before elution at $400 \mathrm{mM}$ imidazole. Purified protein were then dialysed (MWCO 10,000) into 2 litres of $20 \mathrm{mM}$ HEPES pH 7.5, $100 \mathrm{mM} \mathrm{NaCl}$ (Buffer A) at $4^{\circ} \mathrm{C}$ for 6 hours. The enzymes were found to be soluble and active in a range of standard buffers including HEPES ( $\mathrm{pH}$ 7.5) and Tris-HCL pH 8.0-9.5. Additionally, all enzymes were stable for long-term storage at $-80^{\circ} \mathrm{C}$ with $15 \%(\mathrm{v} / \mathrm{v})$ glycerol.

\section{Chemical synthesis of HBA}


564 HBA, also referred to as 4-(4-hydroxyphenyl)-buten-2-one was synthesised by a

565 crossed aldol condensation as previously described ${ }^{62}$. Further details and NMR spectra (Supplementary Figure S10) are provided in the supplementary data.

\section{LC-MS of raspberry ketone and pathway intermediates}

$56950 \mu \mathrm{L}$ samples of time-course reaction in triplicate were removed and inactivated with $450 \mu \mathrm{L}$ of $1 \% \mathrm{HCl}$. Samples were centrifuged at 13,000 rpm for $10 \mathrm{~min}$ at room temperature. The supernatant was directly analysed by liquid-chromatography mass spectrometry (LC-MS), performed with an Agilent 1290 Infinity system with an online diode array detector in combination with a Bruker 6500 quadruple time-of-flight (QToF) mass spectrometer. An Agilent Extend-C18 $2.1 \times 50 \mathrm{~mm}(1.8 \mu \mathrm{m}$ particle size) column was used at a temperature of $25^{\circ} \mathrm{C}$ with a buffer flow rate of $0.2 \mathrm{ml}^{-1} \mathrm{~min}^{-1}$. LC was performed with a linear gradient of buffer $A(0.1 \%$ formic acid) and buffer $B$ $(0.1 \%$ formic acid in acetonitrile). Separation was achieved using $5 \%$ buffer $B$ for 2 min, followed by a linear gradient to $50 \%$ buffer B from 2-9 min, which was held at $50 \%$ buffer B from 9-10 min. Spectra were recorded between a mass range of 90$1000 \mathrm{~m} / \mathrm{z}$ at a rate of 3 spectra per second. Standards were prepared and calibration curves for the intermediates tyrosine, $p$-coumaric acid, HBA and raspberry ketone were derived. Quantitation was based on the MS peak area of precursor or fragment ion in comparison to the analytical standards. Under the conditions used, raspberry ketone is detected as a sodium adduct $\left[\mathrm{M}+\mathrm{Na}^{+}\right]^{+}$or as a diagnostic fragment ion at $m / z=107.49$, corresponding to $\mathrm{C}_{7} \mathrm{H}_{7} \mathrm{O}$. For the standards in solvent, good linearity $\left(R^{2}>0.99\right)$ was achieved over the range of 0.3 to 30 pmol on column. The lower limit of quantitation was set at 0.3 pmol. Samples that were below this limit were repeated by increasing the injection volume to $1 \mu \mathrm{l}$. Due to a lack of a analytical standard and poor separation, $p$-coumoryl-CoA was not quantified.

\section{Enzyme kinetics}

RKS and mutants were purified to homogeneity using nickel IMAC and buffer exchanged into Buffer A. Steady-state kinetics were monitored on a Clariostar (BMG Lifetech) plate-reader monitoring absorbance at $340 \mathrm{~nm}$ following the reduction of $\mathrm{NAD}(\mathrm{P}) \mathrm{H}$ to $\mathrm{NAD}(\mathrm{P})^{+}$with either 4-hydroxyphenyl-3-butan-2-one 596 (hydroxybenzaldehyde) or phenyl-3-butan-2-one (benzaldehyde) as substrates. Assays were performed in triplicate at $30^{\circ} \mathrm{C}$ in $0.1 \mathrm{M}$ potassium phosphate $\mathrm{pH}$ 6.4.

\section{RKS crystallisation and structure determination}

IMAC purified RKS was dialysed for 4 hours in $20 \mathrm{mM}$ Tris-HCL pH 8.0 and $200 \mathrm{mM}$ $\mathrm{NaCl}$. Pure fraction concentrated with a 10,000 MWCO centrifugation concentrator (Amicon) and then run on analytical gel filtration in the same buffer. Purity was assessed by SDS-PAGE and the concentration was determined by $A_{280}$ measurement using an extinction coefficient of $44,030 \mathrm{M}^{-1} \mathrm{~cm}^{-1}$. RKS was concentrated to $10 \mathrm{mg} \mathrm{mL}^{-1}$ and screened in a range of crystallisation conditions using $300 \mathrm{~nL}$ drops containing either a ratio of 1:2 or 2:1 of protein and reservoir buffer. Crystals of $\mathrm{N}$-terminally $\mathrm{His}_{6}$-tagged $\mathrm{RKS}$ were obtained by sitting drop vapour diffusion at $20^{\circ} \mathrm{C}$ after $\sim 3$ days of incubation in $0.1 \mathrm{M}$ MES/imidazole $\mathrm{pH} 6.3$, 11\% (w/v) PEG $550 \mathrm{MME}$ and 5\% (w/v) PEG 20K, $20 \mathrm{mM}$ of amino acid mixture (Molecular dimensions Morpheus system) with $1 \mathrm{mM} \mathrm{NADPH.} \mathrm{Single} \mathrm{cube-shaped}$ crystals grew within 1 week. Native crystals with NADPH bound were soaked in cryoprotectant containing $1 \mathrm{mM} \mathrm{HBA}, 1 \mathrm{mM} \mathrm{NADPH}$ and $20 \%$ glycerol. A native 
614 Source, Didcot, Oxfordshire) from a single crystal diffracting up to $\sim 1.5 \AA$. The crystal 615 belonged to space group P1 (Supplementary Table S1). Further details on structure 616 determination are provided in the supplementary text. The atomic coordinates and 617 structure factors (codes: 6EOW for the ternary structure) has been deposited in the 618 Protein Data Bank. 


\section{Acknowledgements}

621 We would like to thank Dr Tobias Erb (Max Planck Institute) for constructive 622 comments on the preliminary data from this project. We would also like to thank Dr 623 Marc Morgan, Dr Inmaculada Pérez-Dorato and Professor Angelika Gründling for 624 project support concerning the crystallography of RKS, and all the staff in the beam 625 line 104 at the Diamond Light Source (Didcot, Oxfordshire, UK) where the 626 crystallographic datasets were collected. We would also like to thank the EPSRC for 627 funding to SM (EP/K038648/1 - Frontier Engineering). 


\section{References}

631 1. Brochado, A. R. et al. Improved vanillin production in baker's yeast through in silico design. Microb. Cell Fact. 9, 84 (2010).

2. Hansen, E. H. et al. De novo biosynthesis of vanillin in fission yeast (Schizosaccharomyces pombe) and baker's yeast (Saccharomyces cerevisiae). Appl. Environ. Microbiol. 75, 2765-74 (2009).

3. Toogood, H. S. et al. Enzymatic Menthol Production: One-Pot Approach Using Engineered Escherichia coli. ACS Synth. Biol. 4, 1112-1123 (2015).

4. Paddon, C. J. et al. High-level semi-synthetic production of the potent antimalarial artemisinin. Nature 496, 528-32 (2013).

5. Thodey, K., Galanie, S. \& Smolke, C. D. A microbial biomanufacturing platform for natural and semisynthetic opioids. Nat. Chem. Biol. 10, 1-10 (2014).

6. Ye, V. M. \& Bhatia, S. K. Metabolic engineering for the production of clinically important molecules: Omega-3 fatty acids, artemisinin, and taxol. Biotechnol. J. 7, 20-33 (2012).

7. Zhang, Y. H. P. Simpler is better: High-yield and potential low-cost biofuels production through cell-free synthetic pathway biotransformation (SyPaB). ACS Catal. 1, 998-1009 (2011).

8. Yu, X., Liu, T., Zhu, F. \& Khosla, C. In vitro reconstitution and steady-state analysis of the fatty acid synthase from Escherichia coli. Proc. Natl. Acad. Sci. 108, 18643-18648 (2011).

9. Bujara, M., Schümperli, M., Billerbeck, S., Heinemann, M. \& Panke, S. biotransformations. Biotechnol. Bioeng. 106, 376-389 (2010).

10. Schultheisz, H. L., Szymczyna, B. R., Scott, L. G. \& Williamson, J. R. 
Enzymatic de novo pyrimidine nucleotide synthesis. J. Am. Chem. Soc. 133, 297-304 (2011).

11. Opgenorth, P. H., Korman, T. P. \& Bowie, J. U. A synthetic biochemistry module for production of bio-based chemicals from glucose. Nat. Chem. Biol. $12,393-5$ (2016).

12. Bujara, M., Heinemann, M. \& Panke, S. Optimization of a blueprint for in vitro glycolysis by metabolic real-time analysis. Nat. Chem. Biol. 7, 271-277 (2011).

13. Dudley, Q. M., Anderson, K. C. \& Jewett, M. C. Cell-Free Mixing of Escherichia coli Crude Extracts to Prototype and Rationally Engineer High-Titer Mevalonate Synthesis. ACS Synth. Biol. 5, 1578-1588 (2016).

14. Roessner, C. a et al. Overexpression in Escherichia coli of 12 vitamin $\mathrm{B}_{12}$ biosynthetic enzymes. Protein expression and purification 6, 155-163 (1995).

15. Zawada, J. F. et al. Microscale to manufacturing scale-up of cell-free cytokine production-a new approach for shortening protein production development timelines. Biotechnol. Bioeng. 108, 1570-1578 (2011).

16. Zorn, H., Fischer-Zorn, M. \& Berger, R. G. A labeling study to elucidate the biosynthesis of 4-(4-hydroxyphenyl)-butan-2-one (Raspberry Ketone) by Nidula niveotomentosa. Appl. Environ. Microbiol. (2003). doi:10.1128/AEM.69.1.367-372.2003

17. Beekwilder, J. et al. Microbial production of natural raspberry ketone. Biotechnol. J. 2, 1270-1279 (2007). production of raspberry ketone in the wine yeast Saccharomyces cerevisiae via pathway engineering and synthetic enzyme fusion. Microb. Cell Fact. 15, 49 (2016). 
680

681

682

683

684

685

686

687

688

689

690

691

692

693

694

695

696

697

698

699

700

701

702

703

704

19. Katsuyama, Y., Matsuzawa, M., Funa, N. \& Horinouchi, S. Production of curcuminoids by Escherichia coli carrying an artificial biosynthesis pathway. Microbiology 154, 2620-2628 (2008).

20. Morita, H. et al. Structural basis for the one-pot formation of the diarylheptanoid scaffold by curcuminoid synthase from Oryza sativa. Proc. Natl. Acad. Sci. U. S. A. (2010). doi:10.1073/pnas.1011499107

21. Wu, J., Zhou, T., Du, G., Zhou, J. \& Chen, J. Modular optimization of heterologous pathways for de novo synthesis of (2S)-Naringenin in Escherichia coli. PLoS One (2014). doi:10.1371/journal.pone.0101492

22. Lim, C. G., Fowler, Z. L., Hueller, T., Schaffer, S. \& Koffas, M. A. G. High-yield resveratrol production in engineered Escherichia coli. Appl. Environ. Microbiol. 77, 3451-3460 (2011).

23. Koo, H. J. et al. Ginger and turmeric expressed sequence tags identify signature genes for rhizome identity and development and the biosynthesis of curcuminoids, gingerols and terpenoids. BMC Plant Biol. 13, 27 (2013).

24. Ramirez-ahumada, C., Timmermann, B. N. \& Gang, D. R. Biosynthesis of curcuminoids and gingerols in turmeric (Curcuma longa) and ginger (Zingiber officinale): Identification of curcuminoid synthase and hydroxycinnamoyl-CoA thioesterases. 67, 2017-2029 (2006).

25. Morita, H. et al. A structure-based mechanism for benzalacetone synthase from Rheum palmatum. Proc. Natl. Acad. Sci. U. S. A. 107, 669-673 (2010).

26. Zha, W., Rubin-Pitel, S. B., Shao, Z. \& Zhao, H. Improving cellular malonylCoA level in Escherichia coli via metabolic engineering. Metab. Eng. 11, 192-8 (2009).

27. Bennett, B. D. et al. Absolute metabolite concentrations and implied enzyme 
active site occupancy in Escherichia coli. Nat. Chem. Biol. 5, 593-599 (2009).

706

707

708

709

710

711

712

713

714

715

716

717

718

719

720

721

722

723

724

725

726

727

728

729

28. Abe, I., Takahashi, Y., Morita, H. \& Noguchi, H. Benzalacetone synthase. A novel polyketide synthase that plays a crucial role in the biosynthesis of phenylbutanones in Rheum palmatum. Eur. J. Biochem. 268, 3354-3359 (2001).

29. Ceroni, F., Algar, R., Stan, G.-B. \& Ellis, T. Quantifying cellular capacity identifies gene expression designs with reduced burden. Nat. Methods 12, 415-418 (2015).

30. Delgado, J. \& Liao, J. C. Determination of Flux Control Coefficients from transient metabolite concentrations. Biochem. J. 282 ( Pt 3, 919-27 (1992).

31. Weaver, L. J. et al. A kinetic-based approach to understanding heterologous mevalonate pathway function in E. coli. Biotechnol. Bioeng. 112, 111-9 (2015).

32. Linster, C. L., Van Schaftingen, E. \& Hanson, A. D. Metabolite damage and its repair or pre-emption. Nat. Chem. Biol. 9, 72-80 (2013).

33. Davidi, D. et al. Global characterization of in vivo enzyme catalytic rates and their correspondence to in vitro kcat measurements. Proc. Natl. Acad. Sci. U. S. A. 113, 3401-6 (2016).

34. Poppe, L. \& Rétey, J. Friedel-Crafts-type mechanism for the enzymatic elimination of ammonia from histidine and phenylalanine. Angew. Chem. Int. Ed. Engl. 44, 3668-88 (2005).

35. Stuible, H. P., Büttner, D., Ehlting, J., Hahlbrock, K. \& Kombrink, E. Mutational analysis of 4-coumarate:CoA ligase identifies functionally important amino acids and verifies its close relationship to other adenylate-forming enzymes. FEBS Lett. (2000). doi:10.1016/S0014-5793(00)01133-9

36. Koeduka, T. et al. Characterization of raspberry ketone/zingerone synthase, 
catalyzing the alpha, beta-hydrogenation of phenylbutenones in raspberry fruits. Biochem. Biophys. Res. Commun. (2011).

37. Watts, K. T., Mijts, B. N., Lee, P. C., Manning, A. J. \& Schmidt-Dannert, C. Discovery of a Substrate Selectivity Switch in Tyrosine Ammonia-Lyase, a Member of the Aromatic Amino Acid Lyase Family. Chem. Biol. 13, 1317-1326 (2006).

38. Schneider, K. et al. The substrate specificity-determining amino acid code of 4coumarate:CoA ligase. Proc. Natl. Acad. Sci. U. S. A. 100, 8601-6 (2003).

39. McCrate, O. a, Zhou, X. \& Cegelski, L. Curcumin as an amyloid- indicator dye in E. coli. Chem. Commun. 49, 4193-4195 (2013).

40. Katsuyama, Y., Matsuzawa, M., Funa, N. \& Horinouchi, S. In vitro synthesis of curcuminoids by type III polyketide synthase from Oryza sativa. J. Biol. Chem. 282, 37702-37709 (2007).

41. Hassaninasab, A., Hashimoto, Y., Tomita-Yokotani, K. \& Kobayashi, M. Discovery of the curcumin metabolic pathway involving a unique enzyme in an intestinal microorganism. Proc. Natl. Acad. Sci. U. S. A. 108, 6615-20 (2011).

42. Kelley, L. A., Mezulis, S., Yates, C. M., Wass, M. N. \& Sternberg, M. J. E. The Phyre2 web portal for protein modeling, prediction and analysis. Nat. Protoc. 10, 845-58 (2015).

43. Abe, I., Sano, Y., Takahashi, Y. \& Noguchi, H. Site-directed mutagenesis of benzalacetone synthase: The role of PHE 215 in plant type III polyketide synthases. J. Biol. Chem. 278, 25218-25226 (2003).

44. Honda, K. et al. In vitro metabolic engineering for the salvage synthesis of $\mathrm{NAD}^{+}$. Metab. Eng. 35, 114-120 (2016). 
45. Ansell, R. J. \& Lowe, C. R. Artificial redox coenzymes: biomimetic analogues of $\mathrm{NAD}^{+}$. Appl. Environ. Microbiol. 51, 703-710 (1999).

46. Mansell, D. J. et al. Biocatalytic Asymmetric Alkene Reduction: Crystal Structure and Characterization of a Double Bond Reductase from Nicotiana tabacum. ACS Catal. 3, 370-379 (2013).

47. Liu, X. et al. Structure-guided engineering of Lactococcus lactis alcohol dehydrogenase LIAdhA for improved conversion of isobutyraldehyde to isobutanol. J. Biotechnol. 164, 188-195 (2012).

48. Brustad, E. M. \& Arnold, F. H. Optimizing non-natural protein function with directed evolution. Curr. Opin. Chem. Biol. 15, 201-210 (2011).

49. Jarboe, L. R., Liu, P., Kautharapu, K. B. \& Ingram, L. O. Optimization of enzyme parameters for fermentative production of biorenewable fuels and chemicals. Comput. Struct. Biotechnol. J. 3, (2012).

50. Bastian, S. et al. Engineered ketol-acid reductoisomerase and alcohol dehydrogenase enable anaerobic 2-methylpropan-1-ol production at theoretical yield in Escherichia coli. Metab. Eng. 13, 345-352 (2011).

51. Scrutton, N. S., Berry, a \& Perham, R. N. Redesign of the coenzyme specificity of a dehydrogenase by protein engineering. Nature $343,38-43$ (1990).

52. Woodyer, R., Van der Donk, W. A. \& Zhao, H. Relaxing the nicotinamide cofactor specificity of phosphite dehydrogenase by rational design. Biochemistry (2003). doi:10.1021/bi035018b

53. Youn, B. et al. Mechanistic and structural studies of apoform, binary, and ternary complexes of the Arabidopsis alkenal double bond reductase At5g16970. J. Biol. Chem. (2006). doi:10.1074/jbc.M605900200 
54. Paul, S. \& Gupta, M. A Simple and Efficient Method for Selective Single Aldol Condensation Between Arylaldehydes and Acetone. Synth. Commun. 35, 213-222 (2005).

55. Johannes, T. W., Woodyer, R. D. \& Zhao, H. Directed evolution of a thermostable phosphite dehydrogenase for $\mathrm{NAD}(\mathrm{P}) \mathrm{H}$ regeneration. Appl. Environ. Microbiol. 71, 5728-34 (2005).

56. Schwander, T., Schada von Borzyskowski, L., Burgener, S., Cortina, N. S. \& Erb, T. J. A synthetic pathway for the fixation of carbon dioxide in vitro. Science. 354, 900-904 (2016).

57. Hold, C., Billerbeck, S. \& Panke, S. Forward design of a complex enzyme cascade reaction. Nat. Commun. 7, 12971 (2016).

58. Smith, L. R. Rheosmin ('Raspberry Ketone') and Zingerone, and Their Preparation by Crossed Aldol-Catalytic Hydrogenation Sequences. Chem. Educ. 1, 1-18 (1996).

59. Priefert, H., Rabenhorst, J. \& Steinbüchel, A. Biotechnological production of vanillin. Appl. Microbiol. Biotechnol. 56, 296-314 (2001).

60. Moore, S. J. et al. EcoFlex: A Multifunctional MoClo Kit for E. coli Synthetic Biology. ACS Synth. Biol. 5, 1059-1069 (2016).

61. Crosby, H. a., Rank, K. C., Rayment, I. \& Escalante-Semerena, J. C.

Structure-guided expansion of the substrate range of methylmalonyl coenzyme a synthetase (MatB) of Rhodopseudomonas palustris. Appl. Environ. Microbiol. 78, 6619-6629 (2012).

62. Ramachandra, M. S. \& Subbaraju, G. V. Synthesis and bioactivity of novel caffeic acid esters from Zuccagnia punctata. J. Asian Nat. Prod. Res. 8, 683688 (2006). 
A

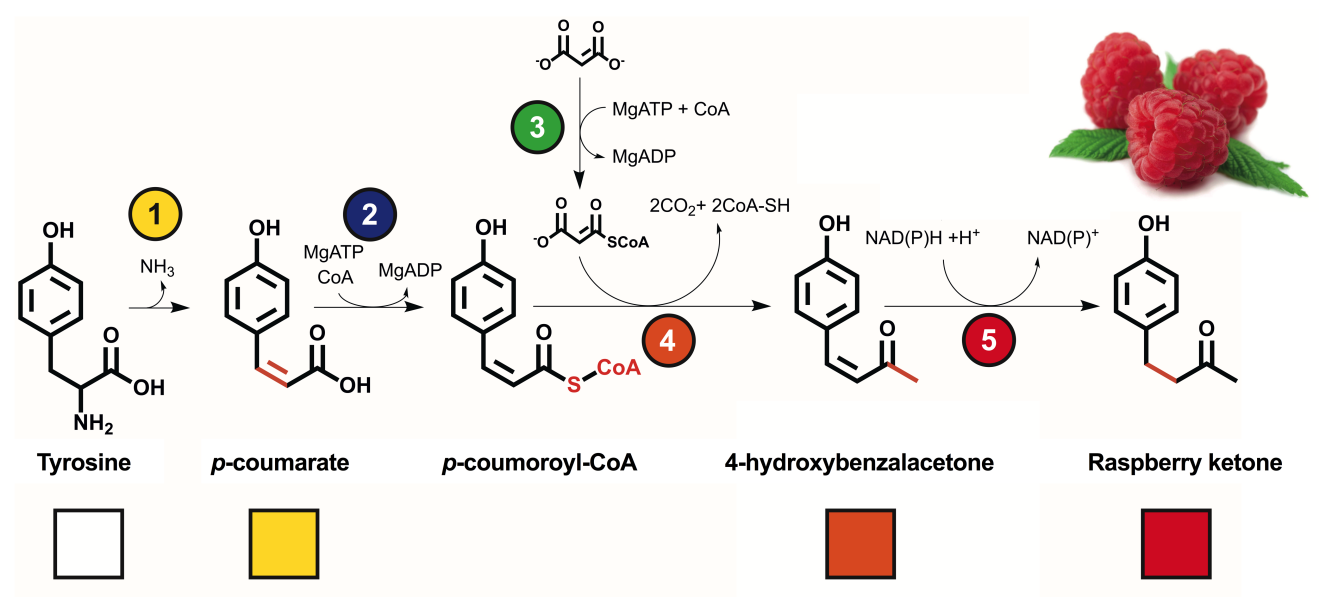

B

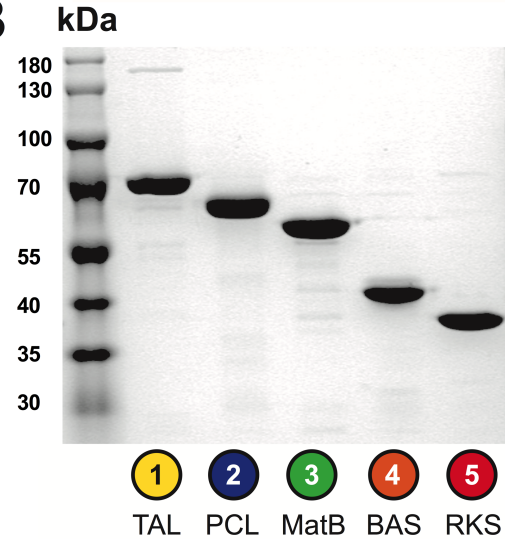

C

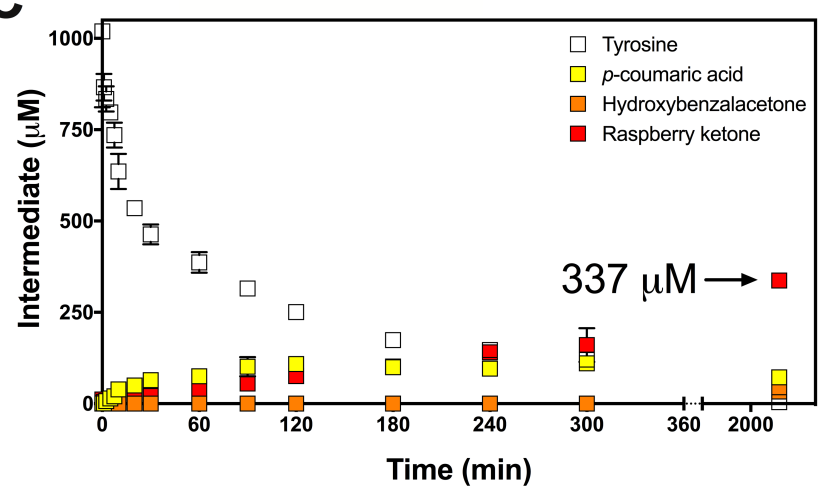

807 Figure 1. A synthetic biochemistry module for raspberry ketone synthesis in vitro. (A) 808 A synthetic pathway for raspberry ketone synthesis from Tyrosine using malonate for 809 malonyl-CoA synthesis. Intermediates quantified by LC-MS include Tyrosine (white 810 box), $p$-coumarate (yellow box), hydroxyHBA (orange box) and raspberry ketone (red 811 box). (B) $2 \mu \mathrm{g}$ of purified enzyme was loaded and analysed by $12 \%$ SDS-PAGE and 812 Coomassie Blue staining. Sizes of $\mathrm{His}_{6}$-tagged recombinant enzyme - $R$. glutinis TAL 813 (77.0 kDa), A. thaliana PCL $(63.2 \mathrm{kDa})$, R. palustris MatB (56.6 kDa), R. palmatum 814 BAS (44.4 kDa), R. rubrum RKS (40.7 kDa). (C) One-pot synthesis of raspberry 815 ketone with $2.5 \mu \mathrm{M}$ of enzymes. For full details, please refer to Supplementary Table 816 S2. 
A.

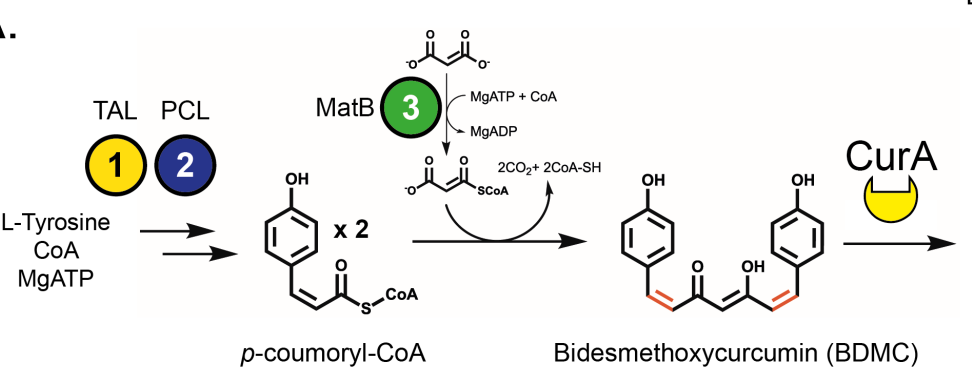

B.
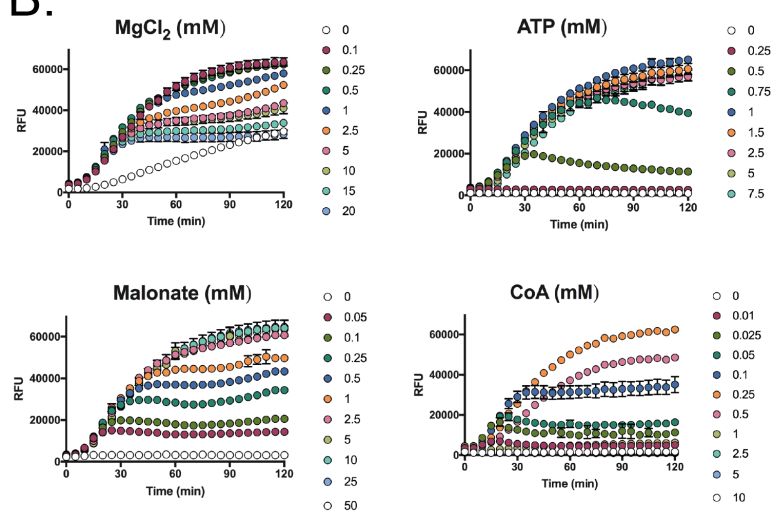

C.

Exc - $425 \mathrm{~nm}$ $\approx$
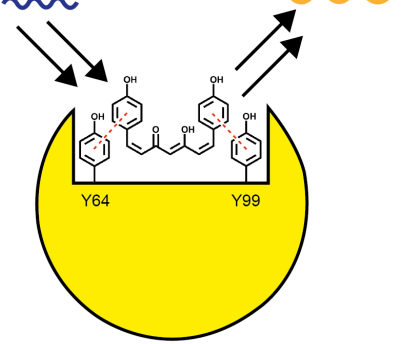

E.
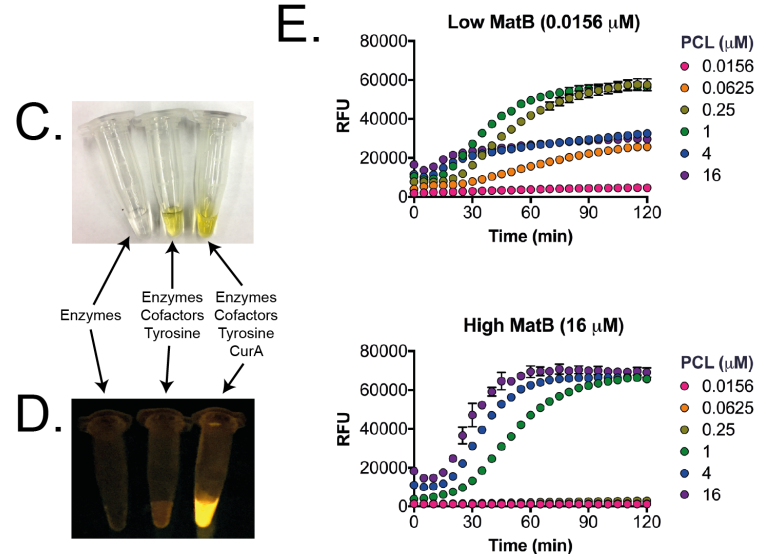

Figure 2. Development of a fluorescence sensor for type III polyketide synthesis. (A) A synthetic pathway for detection of BDMC synthesis from malonyl-CoA and $p$ coumoroyl-CoA. CurA a BDMC/curcumin NADPH-dependent reductase from $E$. coli is used in the absence of NADPH to bind to BDMC/curcumin generating an unique fluorescent output for relative quantitation of pathway activity. (B) Optimisation of cofactors and substrates for type III polyketide synthesis. (C) Visual and (D) fluorescence of in vitro BDMC reactions, with negative controls. (E) Enzyme competition between MatB and PCL, which share CoA and ATP for activity. 
A

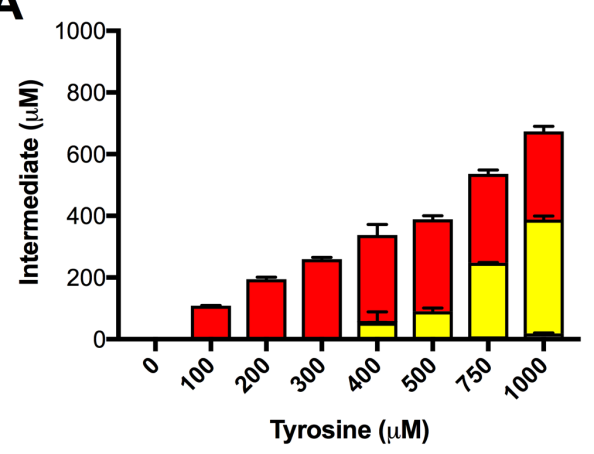

B

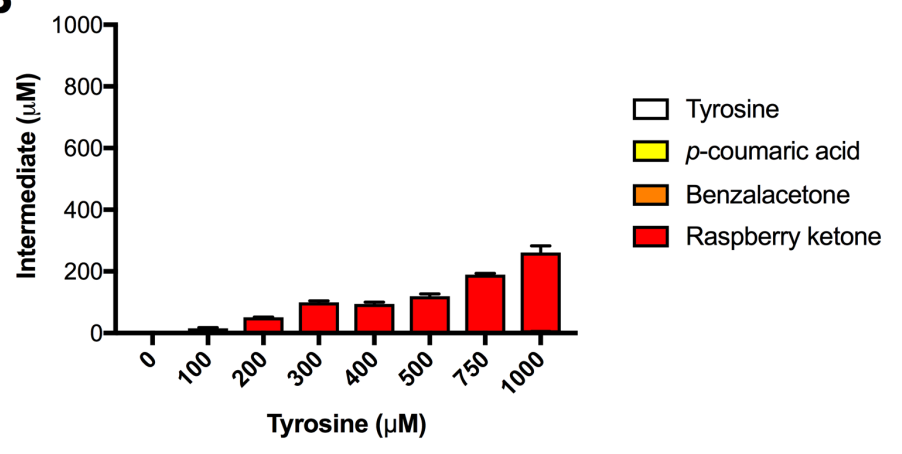

Figure 3. One-pot synthesis of raspberry ketone with a varying concentration of tyrosine. (A) Standard conditions and (B) optimised conditions as outlined in 831 Supplementary Table S2. 

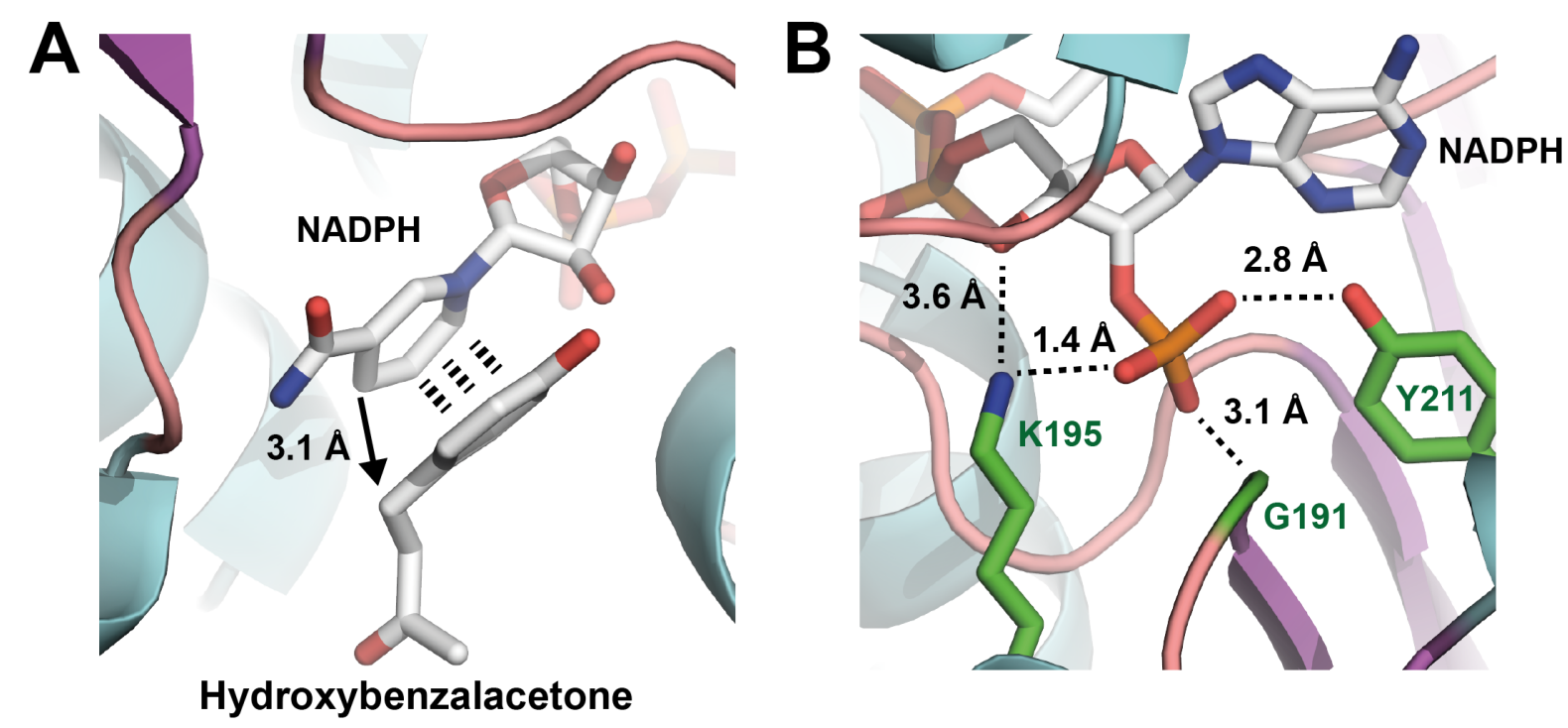

C

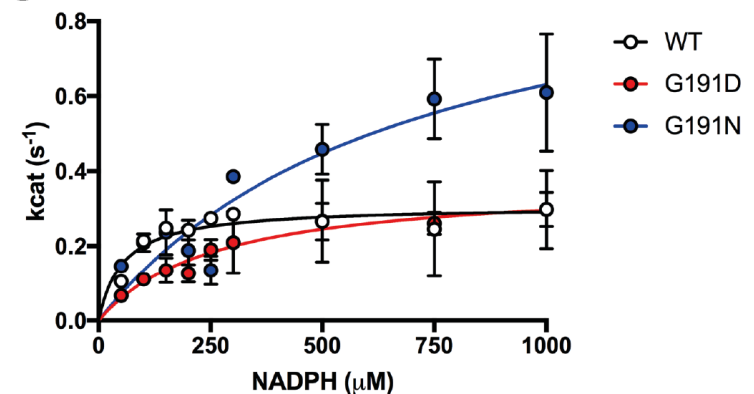

D

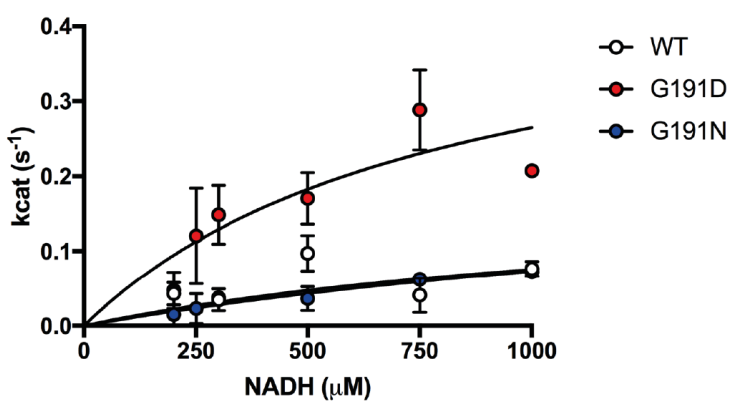

Figure 4. Structure based engineering of the RKS reductase towards NADH utilisation (pdb: 6EOW). (A) Binding site for HBA in proximity to the NADPH cofactor. (B) Cofactor specificity is provided by a triad of binding residues G191, K195 and Y211 with hydrogen bonding to the 5'-ribose phosphate. (C) Kinetic characterisation with NADPH and RKS variants and with (D) NADH as outlined in materials and methods. 


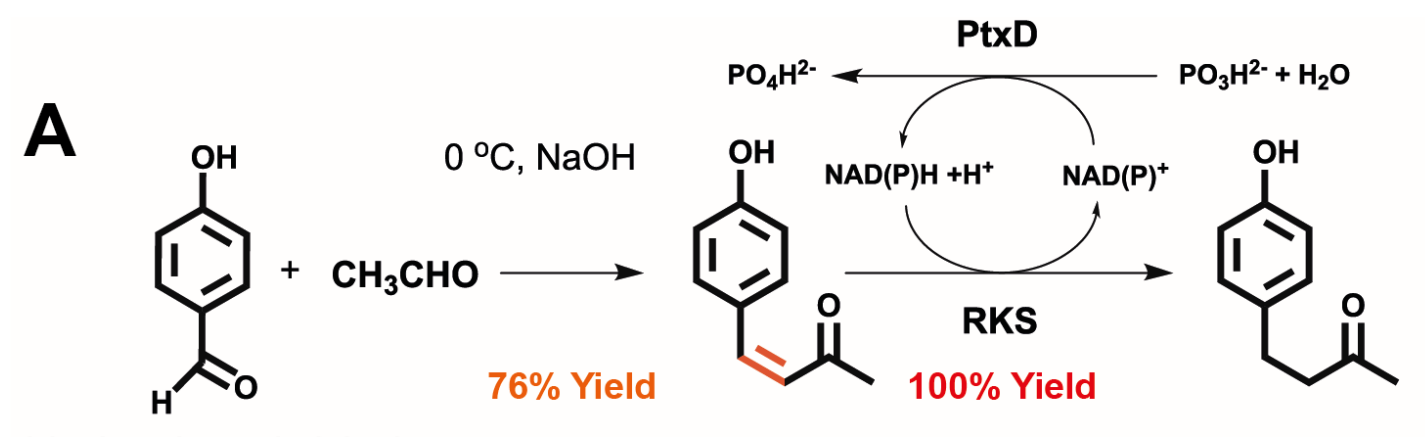

4-hydroxybenzaladehyde

Hydroxybenzalacetone

Raspberry ketone

841

842

843

844

845

846

847

848

849

850

851

852

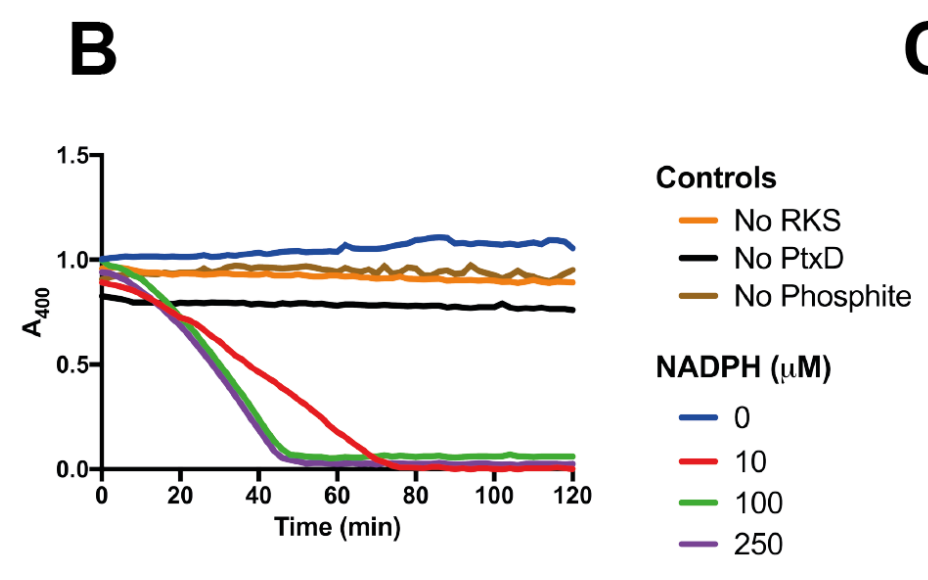

Figure 5. A two-step semi-synthetic route to high-yield raspberry ketone synthesis with NADH and cofactor regeneration. (A) A semi-synthetic pathway for raspberry ketone using aldol condensation and RKS reductase activity with cofactor regeneration. (B) Activity of the thermostable PtxD opt12 phosphite dehydrogenase with $1 \mathrm{mM}$ HBA and $10 \mu \mathrm{M}$ RKS. Negative controls (no enzyme or phosphite) are shown along with a variable concentration of NADPH. (C) Time-course reaction monitoring loss of absorbance at $400 \mathrm{~nm}$ showing reduction of HBA to raspberry ketone. Injections of $1 \mathrm{mM}$ HBA were added in 60 min cycles. An excess of PtxD and $20 \mathrm{mM}$ phosphite was incubated at $30^{\circ} \mathrm{C}$ with $10 \mu \mathrm{M}$ RKS (top panel) or the D191 variant (bottom panel), with either $0.25 \mu \mathrm{M}$ NADPH (blue line) or NADH (red line). 
A

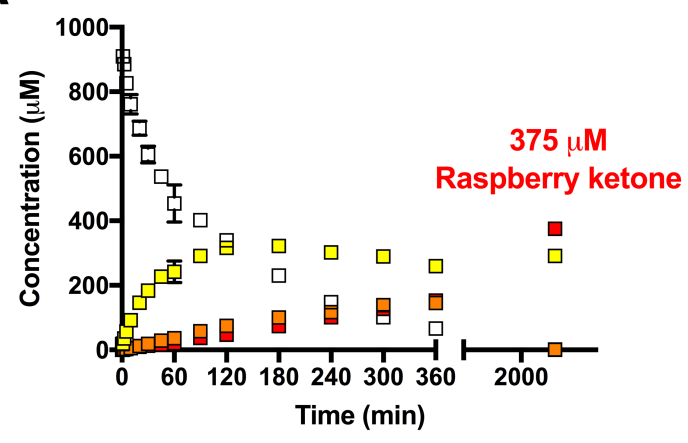

B

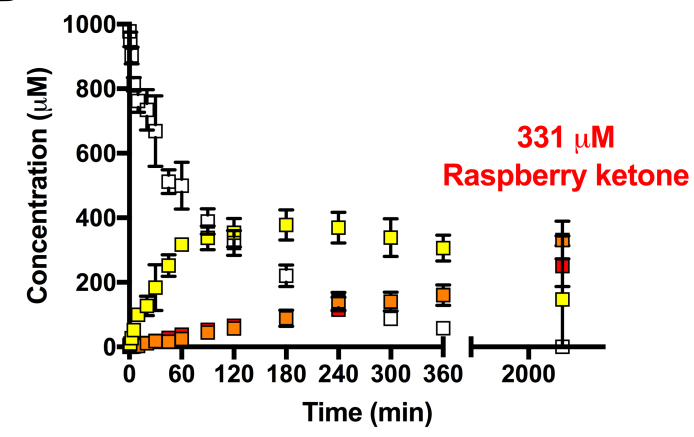

854 Figure 6. One-pot synthesis of raspberry ketone under optimised enzyme levels and cofactor regeneration. (A) Reaction with RKS ${ }^{W T}$ and NADPH. (B) Reaction with $856 \mathrm{RKS}^{\mathrm{D} 191}$ and NADH. Full synthesis conditions are provided in Supplementary Table 857 S2. 